$\nabla$

\section{Toxic epidermal necrosis induced by carbamazepine embedded in the subcutis*}

Jian-Jun $\mathrm{Liu}^{1}$

Shi-Chao $\mathrm{Lu}^{1}$

Jun-Lian Liu ${ }^{1}$

He-Ming Yang ${ }^{2}$

DOI: http:/ / dx.doi.org/10.1590/abd1806-4841.20187725

\section{Dear Editor}

Toxic epidermal necrolysis (TEN) is a rare but life-threatening severe cutaneous adverse reaction with a mortality rate of $25-35 \%$. Herein, we present the case of a 38-year-old woman with a history of epilepsy that developed TEN from carbamazepine embedded in the subcutis. To our knowledge, TEN induced by drugs embedded in the subcutis has never been previously reported in the literature.

A 38-year-old woman with a history of epilepsy was administered subcutaneous carbamazepine in the upper arms at a small clinic. Four days later, a purplish macular rash appeared over the patient's trunk. On admission to hospital, the patient presented with a temperature of $38.2^{\circ} \mathrm{C}$, without localized signs of infection. The erythematous lesions extended over the face and extremities and became confluent. Blisters and epidermal necrosis were present (Figure 1). Multiple mucosal surfaces, including the mouth and genitalia, were also affected. Her lips were covered with erosions and a hemorrhagic crust. Nikolsky's sign was positive. She denied allergies to any medications and had no history of previous carbamazepine exposure. Laboratory studies were normal except for a slightly altered high-sensitivity C-reactive protein of $4.47 \mathrm{mg} / \mathrm{L}$ (normal range, $0-3.0 \mathrm{mg} / \mathrm{L}$ ). A skin biopsy revealed necrosis of keratinocytes throughout the epidermis and subepidermal vesiculation (Figure 2).

Received 07 October 2017.

Accepted 15 December 2017.

* Work conducted at the 306 Hospital of PLA, Beijing, China.

Financial support: None.

Conflict of interests: None.

Department of Dermatology, 306 Hospital of PLA, Beijing, China.

Department of General Surgery, 306 Hospital of PLA, Beijing, China.

MAILING AdDRESS:

Jian-Jun Liu

E-mail: liujianjun306@126.com

(C2018 by Anais Brasileiros de Dermatologia

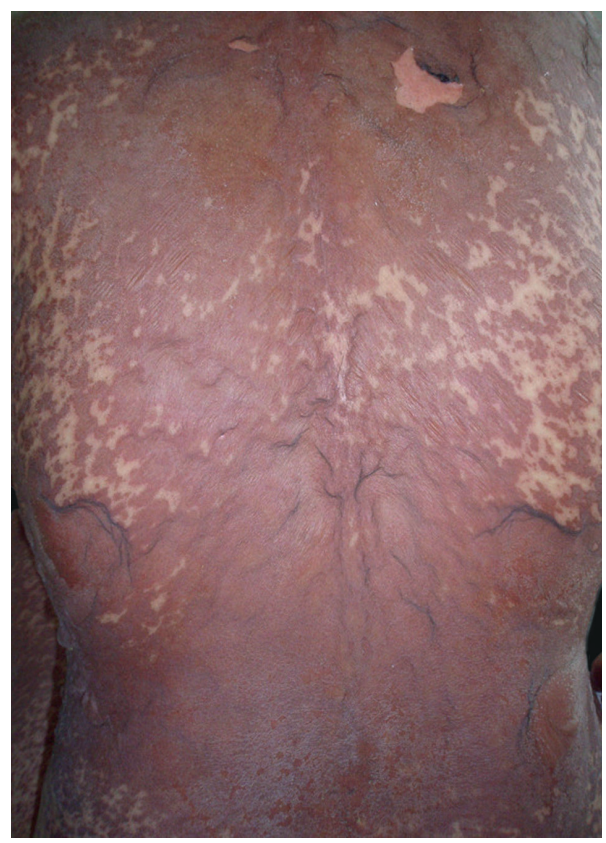

Figure 1:

Epidermal

detachment and confluent blisters on the trunk

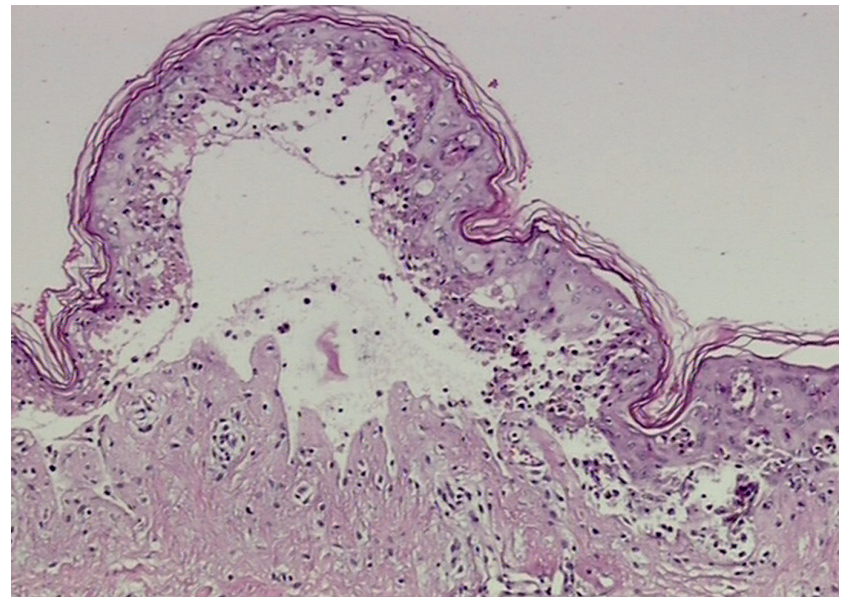

FIgURE 2: Histological examination showing necrosis of keratinocytes throughout the epidermis, and subepidermal vesiculation (Hematoxylin \& eosin, x200)

In this case, the diagnosis of TEN was based on medical history, clinical features, and the result of a skin biopsy. The patient was treated with extensive surgical debridement at the site of the embedded drug, which helped to clear out any residual drug (Figure 3). At the same time, high-dose intravenous methylprednisolone $(80 \mathrm{mg} / \mathrm{d})$ and immunoglobulin $(2.1 \mathrm{~g} / \mathrm{kg})$ were administered. In the following days, the wounds were irrigated daily with hydrogen peroxide and saline, and then packed with Vaseline gauze. Mucocutaneous erosions were treated with infrared radiation, followed by multiple applications of antibiotic ointments. After one month, the patient's lesions lesions healed with post-inflammatory hyperpigmentation in some of the affected areas. 


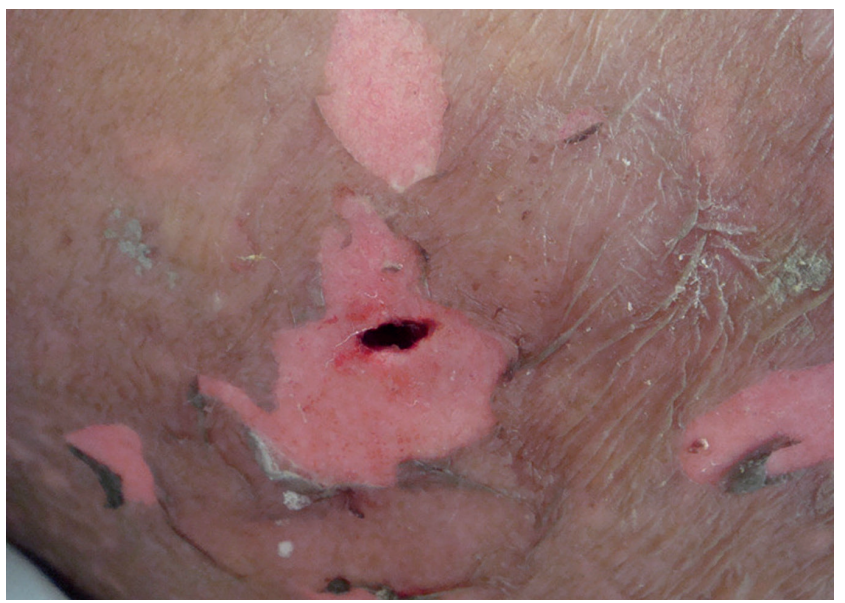

Figure 3: The wound on the upper arm after debridement, measuring approximately $15 \times 5 \mathrm{~mm}$

TEN is an acute life-threatening mucocutaneous disease that involves epidermal detachment of $>30 \%$ of the body surface area. Drug reactions are responsible for $80 \%$ to $95 \%$ of TEN cases. Carbamazepine causes Stevens-Johnson syndrome (SJS)/TEN at a frequency of 14 per 100,000 users. ${ }^{1}$

The pathogenesis of TEN remains unknown, but multiple inflammatory mediators have been implicated, including soluble Fas ligand (sFasL), tumor necrosis factor alpha (TNF- $\alpha$ ) and granzyme B/perforin, as well as granulysin, which appears to be the pivotal mediator of keratinocyte apoptosis. ${ }^{2}$ Recent advances in pharmacogenomic studies have provided evidence for genetic predispositions to SJS/TEN. The association between HLA-B*1502 and carbamazepine-induced SJS/TEN has been validated in Han-Chinese, Thai, and Malaysian populations..$^{3-5}$

Currently, no treatment modality has been established as standard for patients with TEN. Obviously, presumptive causative drugs should be stopped as soon as possible. In this case of subcutis drug-induced TEN, we initiated treatment with extensive surgical debridement of the affected area. Additionally, aggressive nutritional support and appropriate cutaneous care are also vitally important. Although the role of corticosteroids in TEN remains controversial, we observed the efficacy and safety of corticosteroids and immunoglobulin administered at an early stage of TEN in our patient.

This case highlights the importance of increased awareness of TEN and the need for clinicians to exercise caution when prescribing carbamazepine. Screening for genetic markers before prescribing carbamazepine can help prevent the occurrence of TEN.

\section{REFERENCES}

1. Roujeau JC, Stern RS. Severe adverse cutaneous reactions to drugs. N Engl J Med. 1994;331:1272-85.

2. Chung WH, Hung SI, Yang JY, Su SC, Huang SP, Wei CY, et al. Granulysin is a key mediator for disseminated keratinocyte death in Stevens-Johnson syndrome and toxic epidermal necrolysis. Nat Med. 2008;14:1343-50.

3. Zhang Y, Wang J, Zhao LM, Peng W, Shen GQ, Xue L, et al. Strong association between HLA-B*1502 and carbamazepine-induced Stevens-Johnson syndrome and toxic epidermal necrolysis in mainland Han Chinese patients. Eur J Clin Pharmacol. 2011;67:885-7.

4. Tassaneeyakul W, Tiamkao S, Jantararoungtong T, Chen P, Lin SY, Chen WH, et al. Association between $\mathrm{HLA}-\mathrm{B} * 1502$ and carbamazepine-induced severe cutaneous adverse drug reactions in a Thai population. Epilepsia. 2010:51:926-30.

5. Chang CC, Too CL, Murad S, Hussein SH. Association of HLA-B*1502 allele with carbamazepine-induced toxic epidermal necrolysis and Stevens-Johnson syndrome in the multi-ethnic Malaysian population. Int J Dermatol. 2011;50:221-4.

\section{AUTHORS CONTRIBUTION}

Jian-jun Liu

ID ORCID 0000-0002-8743-1772

Approval of the final version of the manuscript; Elaboration and writing of the manuscript; Obtaining, analyzing and interpreting the data; Effective participation in research orientation; Intellectual participation in propaedeutic and/or therapeutic conduct of cases studied; Critical review of the literature; Critical review of the manuscript

Shi-chao Lu

ORCID 0000-0003-2711-8765

Approval of the final version of the manuscript; Elaboration and writing of the manuscript; Intellectual participation in propaedeutic and/or therapeutic conduct of cases studied; Critical review of the literature; Critical review of the manuscript

Jun-lian Liu

ORCID 0000-0003-4482-4645

Approval of the final version of the manuscript; Intellectual participation in propaedeutic and/or therapeutic conduct of cases studied; Critical review of the literature; Critical review of the manuscript

He-ming Yang

ORCID 0000-0002-2867-6809

Approval of the final version of the manuscript; Intellectual participation in propaedeutic and/or therapeutic conduct of cases studied; Critical review of the manuscript

How to cite this article: Liu JJ, Lu SC, Liu JL, Yang HM. Toxic epidermal necrosis induced by carbamazepine embedded in the subcutis. An Bras Dermatol. 2018;93(4):620-1. 